\title{
Cardiovascular Risk Assessment Based on Combined Body Mass Index and Waist Circumference in Korean Adults
}

\author{
Young Gyu Cho \\ Department of Family Medicine, Inje Universtiy Seoul-Paik Hospital, Inje University College of Medicine, Seoul, Korea
}

Obesity is a state of excessive accumulation of body fat and is associated with increased cardiovascular risk. Traditionally, body mass index (BMI) has been used as a measure for assessing health risk due to body fatness. However, BMI has been shown to have a limitation in that it does not differentiate body fat mass from lean body mass. In persons with an unusual body fat mass-to-lean body mass ratio, such as athletes and the elderly, BMI may lead to bias when used in health risk assessment. ${ }^{1)}$ Another limitation of BMI is the fact that it does not provide any information on body fat distribution. Many studies have shown that different patterns of regional fat deposition have different metabolic effects. ${ }^{2)}$ Intraabdominal or visceral fat has been reported to be more strongly associated with an increased risk for cardiovascular disease than subcutaneous fat. ${ }^{3)}$ The guidelines recommended that abdominal adiposity should be assessed in conjunction with overall adiposity to identify persons at risk. Waist circumference (WC) is a simple and practical indicator of abdominal adiposity. ${ }^{4)}$

Many studies have addressed whether the combination of $\mathrm{BMI}$ and WC is more predictive of cardiovascular risk than either measure alone. Although it is obvious that the addition of WC to BMI improves the predictive ability of BMI for cardiovascular risk, there is controversy about whether the reverse is true. Janssen et al. ${ }^{5}$ investigated whether BMI adds to the predictive power of WC in assessing obesity-related comorbidities such as hypertension, dyslipidemia, and metabolic syndrome in the US population. They reported that when both $\mathrm{BMI}$ and WC were included in the same regression model as continuous variables for predicting comorbidity, WC, and not BMI, was a significant predictor of comorbidity. Czernichow et al. ${ }^{6)}$ examined which index of obesity is the better discriminator of the mortality risk from cardiovascular disease, by conducting a meta-analysis of a series of nine cohort studies in the British general population. In their study, measures of abdominal adiposity (WC and waist-to-hip ratio), but not BMI, were related to an increased risk for cardiovascular disease mortality. Furthermore, the combined measure of BMI and WC did not improve the discrimination capacity for cardiovascular disease mortality. In contrast, Wildman et al. ${ }^{7)}$ showed that persons with higher BMI values had a higher likelihood of having metabolic disorders than those with lower BMI values, even after adjustment for WC in Chinese adults. Hou et al. ${ }^{8)}$ also reported that both WC and BMI adds to the predictive power of each other in identifying cardiovascular disease risk in Chinese adults.

In the present issue, Yoo et al. ${ }^{9)}$ investigated whether the cross-tabulated combination of BMI and WC can assess cardiovascular risk factors better than BMI or WC alone in Korean adults. They obtained their data from the National Health Insurance Service national sample cohort of the years 20122013, which consisted of 328,789 Korean adults (169,011 men and 159,778 women) aged 30 years and older. They showed that higher BMI categories were associated with a greater risk of having cardiovascular risk factors within the same category of WC and vice versa. Their results were consistent with the study results from the Chinese population. ${ }^{7,8)}$ In previous studies based on Asians, although diabetes was more strongly associated with WC than with BMI, hypertension had a stronger association with $\mathrm{BMI}$ than with WC. ${ }^{7,8,10,11)}$ These findings were also observed in the study of Yoo et al. ${ }^{9)} \mathrm{WC}$ is a surrogate marker of visceral adiposity, which is closely associated with 
disturbed glucose metabolism. BMI well reflects body fluid volume, blood viscosity, and cardiac output, which are closely associated with blood pressure level. ${ }^{8,11}$

The combined measure of BMI and WC has clinical benefits compared with either measure alone in assessing cardiovascular risk. It should be kept in mind that the combined BMI and WC measure is a simple and practical tool to identify persons at a risk for developing cardiovascular disease in clinical practice.

\section{CONFLICT OF INTEREST}

No potential conflict of interest relevant to this article is reported.

\section{REFERENCES}

1. Prentice AM, Jebb SA. Beyond body mass index. Obes Rev 2001;2:141-7.

2. Snijder MB, van Dam RM, Visser M, Seidell JC. What aspects of body fat are particularly hazardous and how do we measure them? Int J Epidemiol 2006;35:83-92.

3. Despres JP. Body fat distribution and risk of cardiovascular disease: an update. Circulation 2012;126:1301-13.

4. National Institutes of Health. Clinical guidelines on the identification, evaluation, and treatment of overweight and obesity in adults: the evidence report. Obes Res 1998;6 Suppl 2:51S-209S.

5. Janssen I, Katzmarzyk PT, Ross R. Waist circumference and not body mass index explains obesity-related health risk. Am J Clin Nutr 2004;79:379-84.

6. Czernichow S, Kengne AP, Stamatakis E, Hamer M, Batty GD. Body mass index, waist circumference and waist-hip ratio: which is the better discriminator of cardiovascular disease mortality risk?: evidence from an individual-participant meta-analysis of 82864 participants from nine cohort studies. Obes Rev 2011;12:680-7.

7. Wildman RP, Gu D, Reynolds K, Duan X, Wu X, He J. Are waist circumference and body mass index independently associated with cardiovascular disease risk in Chinese adults? Am J Clin Nutr 2005;82:1195202.

8. Hou X, Lu J, Weng J, Ji L, Shan Z, Liu J, et al. Impact of waist circumference and body mass index on risk of cardiometabolic disorder and cardiovascular disease in Chinese adults: a national diabetes and metabolic disorders survey. PLoS One 2013;8:e57319.

9. Yoo B, Nam H, Hwang IC, Park Y. Correlation of cardiovascular risk factors with central obesity and multiple body mass index in Korea. Korean J Fam Med 2017;38:338-45.

10. Decoda Study Group, Nyamdorj R, Qiao Q, Lam TH, Tuomilehto J, Ho SY, et al. BMI compared with central obesity indicators in relation to diabetes and hypertension in Asians. Obesity (Silver Spring) 2008;16:1622-35.

11. Feng RN, Zhao C, Wang C, Niu YC, Li K, Guo FC, et al. BMI is strongly associated with hypertension, and waist circumference is strongly associated with type 2 diabetes and dyslipidemia, in northern Chinese adults. J Epidemiol 2012;22:317-23. 